\title{
A Systematic Review on Balance Performance in Patients With Bilateral Vestibulopathy
}

Citation for published version (APA):

Herssens, N., Verbecque, E., McCrum, C., Meijer, K., van de Berg, R., Saeys, W., Vereeck, L., Van Rompaey, V., \& Hallemans, A. (2020). A Systematic Review on Balance Performance in Patients With Bilateral Vestibulopathy. Physical Therapy, 100(9), 1582-1594. https://doi.org/10.1093/ptj/pzaa083

Document status and date:

Published: 01/09/2020

DOI:

10.1093/ptj/pzaa083

Document Version:

Publisher's PDF, also known as Version of record

Document license:

Taverne

Please check the document version of this publication:

- A submitted manuscript is the version of the article upon submission and before peer-review. There can be important differences between the submitted version and the official published version of record.

People interested in the research are advised to contact the author for the final version of the publication, or visit the DOI to the publisher's website.

- The final author version and the galley proof are versions of the publication after peer review.

- The final published version features the final layout of the paper including the volume, issue and page numbers.

Link to publication

\footnotetext{
General rights rights.

- You may freely distribute the URL identifying the publication in the public portal. please follow below link for the End User Agreement:

www.umlib.nl/taverne-license

Take down policy

If you believe that this document breaches copyright please contact us at:

repository@maastrichtuniversity.nl

providing details and we will investigate your claim.
}

Copyright and moral rights for the publications made accessible in the public portal are retained by the authors and/or other copyright owners and it is a condition of accessing publications that users recognise and abide by the legal requirements associated with these

- Users may download and print one copy of any publication from the public portal for the purpose of private study or research.

- You may not further distribute the material or use it for any profit-making activity or commercial gain

If the publication is distributed under the terms of Article $25 \mathrm{fa}$ of the Dutch Copyright Act, indicated by the "Taverne" license above, 
N. Herssens, MSc, Department of Rehabilitation Sciences and Physiotherapy/ Movant, Faculty of Medicine and Health Sciences, University of Antwerp,

Universiteitsplein 1, Campus Drie Eiken, DR314, 2610 Wilrijk, Belgium; Multidisciplinary Motor Centre Antwerp ( $\left.\mathrm{M}^{2} \mathrm{OCEAN}\right)$, University of Antwerp, Antwerp, Belgium. Address all correspondence to Mr Herssens at: Nolan. herssens@uantwerpen.be.

E. Verbecque, PhD, Department of Rehabilitation Sciences and Physiotherapy/Movant, Faculty of Medicine and Health Sciences, University of Antwerp; Rehabilitation Research Centre (REVAL),

Rehabilitation Sciences and Physiotherapy, Hasselt University, Diepenbeek, Belgium.

C. McCrum, PhD, Department of Nutrition and Movement Sciences, NUTRIM School of Nutrition and Translational Research in Metabolism, Maastricht University Medical Centre+, Maastricht, the Netherlands; Institute of Movement and Sport Gerontology, German Sport University, Cologne, Germany.

K. Meijer, PhD, Department of Nutrition and Movement Sciences, NUTRIM School of Nutrition and Translational Research in Metabolism, Maastricht University Medical Centre ${ }^{+}$.

R. van de Berg, MD, PhD, Department of Otorhinolaryngology and Head and Neck Surgery, Division of Balance Disorders, Faculty of Health Medicine and Life Sciences, School for Mental Health and Neuroscience, Maastricht University Medical Centre+; Faculty of Physics, Tomsk State University, Tomsk, Russia.

W. Saeys, PhD, Department of Rehabilitation Sciences and Physiotherapy/Movant, Faculty of Medicine and Health Science, University of Antwerp; RevArte Rehabilitation Hospital, Edegem, Antwerp.

\section{A Systematic Review on Balance Performance in Patients With Bilateral Vestibulopathy}

Nolan Herssens, Evi Verbecque, Christopher McCrum, Kenneth Meijer, Raymond van de Berg, Wim Saeys, Luc Vereeck, Vincent Van Rompaey, Ann Hallemans

Objective. Patients with bilateral vestibulopathy (BVP) have severe balance deficits, but it is unclear which balance measures are best suited to quantify their deficits and approximate the diversity of their self-reports. The purpose of this study was to explore measures of balance control for quantifying the performance of patients with BVP related to different balance domains, allowing targeted assessment of response to intervention.

Methods. MEDLINE, Web of Science, and Embase were systematically searched on October 9, 2019. The Scottish Intercollegiate Guidelines Network checklist for case-control studies was applied to assess each individual study's risk of bias. Standardized mean differences (SMD) were calculated based on the extracted numeric data and reported according to the type of sensory perturbation in the balance tasks.

Results. Twelve studies (1.3\%) met the eligibility criteria and were analyzed, including data of 176 patients with BVP, 196 patients with unilateral vestibulopathy, and 205 healthy controls between 18 and 92 years old. In general, patients with BVP were either unable to maintain (or had reduced) balance during tasks with multisensory perturbations compared with healthy controls (range of mean SMD $=1.52-6.92$ ) and patients with unilateral vestibulopathy (range of absolute mean SMD $=0.86-1.66$ ).

Conclusions. During clinical assessment to quantify balance control in patients with BVP, tasks involving multisensory perturbations should be implemented in the test protocol.

Impact. As patients with BVP show difficulties with movement strategies, control of dynamics, orientation in space, and cognitive processing, clinicians should implement these aspects of balance control in their assessment protocol to fully comprehend the balance deficits in these patients. 
L. Vereeck, PT, PhD, Department of Rehabilitation Sciences and Physiotherapy/Movant, Faculty of Medicine and Health Science, University of Antwerp; Multidisciplinary Motor Centre Antwerp (M ${ }^{2}$ OCEAN), University of Antwerp.

V. Van Rompaey, MD, PhD, Department of Otorhinolaryngology and Head \& Neck Surgery, Antwerp University Hospital, Edegem, Belgium; Department of Translational Neurosciences, Faculty of Medicine and Health Sciences, University of Antwerp

A. Hallemans, PhD, Department of Rehabilitation Sciences and Physiotherapy/Movant, Faculty of Medicine and Health Science, University of Antwerp;

Multidisciplinary Motor Centre Antwerp (M ${ }^{2}$ OCEAN), University of Antwerp.

[Herssens N, Verbecque E, McCrum C, et al. A systematic review on balance performance in patients with bilateral vestibulopathy. Phys Ther. 2020;100:1582-1594.]

(c) The Author(s) 2020. Published by Oxford University Press on behalf of American Physical Therapy

Association.

All rights reserved. For permissions, please e-mail:

journals.permissions@oup.com.

Published Ahead of Print:

May 4, 2020

Accepted: March 1, 2020

Submitted: June 5, 2019
B ilateral vestibulopathy (BVP) is an uncommon (28/100,000 US adults in 2008) ${ }^{1}$ but highly disabling disorder characterized by a partial or complete loss of vestibular function on both sides. ${ }^{2}$ A bilateral loss of vestibular function can be due to a dysfunction of the vestibular end organs, the vestibular nerves, or a combination of both. ${ }^{3,4}$ In 2017, the diagnostic criteria of BVP were published by the Classification Committee of the Bárány Society. ${ }^{5}$ These diagnostic criteria include symptoms of movement-induced blurred vision during walking or quick head movements and unsteadiness during standing and walking that worsens in darkness and on uneven ground. ${ }^{5}$

As head movements aggravate their symptoms, patients with BVP are at risk for limiting their activities of daily life, becoming more sedentary, moving more rigidly to decrease their symptoms, and eventually developing concern for and risk of falling. ${ }^{6}{ }^{\text {These }}$ adverse effects of BVP on function and participation highlight the need for adequate assessment of balance deficits in order to formulate a physical therapy diagnosis to guide targeted intervention. ${ }^{6}$ Such assessments need to take into account a comprehensive, multi-factorial definition of balance where controlling the center of mass in relation to the base of support during self-initiated and externally triggered disturbances is implemented.?

Alongside the defined diagnostic criteria, Strupp et $\mathrm{al}^{5}$ made several suggestions regarding the assessment of the vestibulo-ocular reflex (VOR) and the vestibulo-spinal reflex. To assess the VOR, a large variety of both laboratory and bedside test methods was described. ${ }^{5}$ To address the vestibulo-spinal reflex, on the other hand, only the Romberg test was recommended. ${ }^{5}$ However, this test is limited to standing balance with feet together and with eyes open or closed. Considering the broad range of clinical symptoms reported by patients with BVP, a single test such as the Romberg test seems inadequate, and a more comprehensive approach, taking several task constraints and sensory conditions into account, seems justified.

Balance control is required in both static and dynamic situations, suggesting that its assessment should comprise both task constraints. ${ }^{8}$ Furthermore, balance control is highly influenced by the degree to which sensory information is available. It is well-established that the sensory triad of vestibular-, visual-, and proprioceptive information is crucial to adequately achieve, maintain, and restore balance control during daily activities. ${ }^{8}$ All sensory systems provide accurate information on body motion with respect to (1) surface orientation (proprioception), (2) the visual scene (vision), and (3) gravitational space (vestibular). ${ }^{9}$ Patients with BVP therefore mainly experience postural instability, which increases in darkness or on uneven terrain. ${ }^{2,10}$ These sensory perturbations should therefore be reflected in the assessment protocol.

Vestibular rehabilitation in vestibular patients focuses on adaptation in case of residual function or enabling substitution of the vestibular loss by enhancing the use of visual and proprioceptive information. This requires the selection and weighting of the incoming visual and proprioceptive information to enhance balance control. ${ }^{11}$ The weight attributed to each available sensory input represents the proportion of information that contributes to balance control. ${ }^{9}$ Depending on the situation, task, and goal, the sensory information will be reweighted with the input providing the most accurate information having the highest weight, without excluding information from any of the remaining sensors. ${ }^{12}$ To improve this reweighting process via physical rehabilitation programs, sensitive measurement tools for balance control need to be selected, because this process of sensory substitution is highly variable and depends on individual factors. ${ }^{13,14}$ For example, the degree to which vestibular function is preserved plays a role in this process, but also predisposition of an increased reliance on visual or somatosensory cues in some individuals has been hypothesized..$^{15,16}$ 


\section{Balance Performance in BVP}

To provide suitable vestibular rehabilitation for people with BVP, enhancing sensory reweighting and compensatory strategies and by extension quality of life, more insight into various balance assessment methods is needed. In particular, it is of interest to compare this population with a group of patients with unilateral peripheral vestibular loss and healthy controls (HC) with bilaterally intact vestibular function. By comparing these groups, insights are gained into the degree to which balance performances vary according to vestibular functioning (intact/unilateral hypofunction/bilateral hypofunction). Therefore, the aim of this review is to explore measures of balance control currently used in literature to quantify the performances of patients with BVP on different balance domains, thereby approximating the diversity of their subjective complaints, which could ultimately allow targeted assessment of response to intervention.

\section{Methods}

\section{Protocol and Registration}

This systematic review was conducted according to the Preferred Reporting Items for Systematic Review and Meta-Analysis Statement. The protocol is available online at PROSPERO (www.crd.york.ac.uk/prospero; registration no. CRD42018102354).

\section{Data Sources and Searches}

A systematic search was last performed on October 9, 2019, in PubMed, Web of Science (Web of Knowledge), and Embase using synonyms for the keyword "bilateral vestibulopathy." No filters were applied. Specifics on the search queries for the 3 databases are available in Supplementary Appendix 1. The search query was defined by 2 researchers.

\section{Study Selection}

Relevant studies were identified using predefined selection criteria according to the Population Intervention Comparison Outcome Study design method.

Study design. Original studies written in English, Dutch, French, or German, with a case-control design were considered relevant as well as studies in which norm-referenced (reference data derived from healthy adult participants) assessment tools were applied. Conference proceedings/-reports, editorials, letters, case studies/-series, abstract only, (systematic) reviews, and meta-analyses were excluded.

Population. Adult patients ( $\geq 18$ years old) with isolated BVP were of interest. All diseases or syndromes where BVP occurs concurrently with other symptoms were excluded (eg, cerebellar ataxia with neuropathy and vestibular areflexia syndrome, diabetes mellitus, all types of cerebellar disorders, neuropathies, etc). Children and adolescents were excluded because of the ongoing maturation or developmental influence.
Comparison. Performances on balance tests of patients with BVP had to be compared with either HC adults and/or adult patients with unilateral vestibulopathy (UVP) to determine the influence of bilateral vestibular dysfunction on balance control.

Outcome. Balance control was measured using a standardized assessment tool in any static (ie, timed bipedal stance or posturography), quasi-dynamic (ie, chair stance test), or dynamic (ie, Timed Up and Go [TUG] test or 3-dimensional gait analysis) situation for which numeric data were reported for both patients and controls. Self-reported balance deficits were excluded.

Risk of bias. Studies of poor methodological quality were excluded. Two independent researchers (N.H., E.V.) applied the selection criteria on title and abstract (phase 1) and on full text (phase 2) in the same sequence: design, population, comparison, and outcome. After each phase, the reason for inclusion and exclusion of citations was discussed in a consensus meeting. To ensure that no relevant articles were missed, the references of all studies, included after phase 2 , were screened and included if eligible. The selection process is presented in Supplementary Appendix 2.

\section{Data Extraction and Quality Assessment}

Data extraction. If the study was eligible, the following data were extracted:

- General population characteristics (number of participants per group, mean age and SD, age range, sex distribution) and specific characteristics for the patient groups (UVP/BVP: diagnostic criteria, etiology, and time since the vestibular loss occurred);

- Classification of the balance tasks according to the sensory perturbations: "without sensory perturbations," "perturbations of the base of support," "visual perturbations," "vestibular perturbations," and "more than 1 sensory perturbation." Subsequently, within these classifications, tasks were further classified as "standing" or "walking" tasks. In Supplementary Appendix 3, a flowchart depicts the classification process of the different perturbations;

- Mean and SD for each parameter. In case of median values and interquartile ranges, Hillier and Inglis-Jassiem's formula for SD $(\mathrm{SD}=0.75 \times$ interquartile range) was applied and the median was assumed to equate the mean. ${ }^{17}$

\section{Quality Assessment}

To identify risk of bias in individual studies, the Scottish Intercollegiate Guidelines Network checklist for case-control studies was applied. ${ }^{18,19}$ The checklist contains 2 sections: internal validity and overall quality assessment of the study. In the internal validity section, risk of bias in the selection of participants, assessment, 
confounding, and statistical analysis were assessed. One item was omitted from the risk of bias assessment"Measures will have been taken to prevent knowledge of primary exposure influencing case ascertainment"because loss of balance is one of the primary symptoms in patients with BVP.

Subsequently, the studies' overall quality was rated as high quality $(++)$ when $\geq 8 / 10$ criteria were met, as acceptable quality $(+)$ when $6-7 / 10$ criteria were met, and as low quality (0) when either most criteria were not met $(\geq 5 / 10)$ or significant flaws relating to key aspects of the study design were identified. ${ }^{19}$ Studies were rejected from data extraction if overall quality was low or if the study did not include clear definitions of the source population, comment on how cases were selected, address the influence of confounding factors, or provide a stat-ement on psychometrics of the outcome measures or if the outcome measures of interest were secondary outcomes. All studies were assessed by 2 independent researchers (N.H., E.V.) and results were discussed in a consensus meeting.

\section{Data Synthesis and Analysis}

Standardized mean differences (SMD, mean and 95\% CI) were calculated in Review Manager (RevMan 5.3, Cochrane, London, UK) and presented as forest plots for each type of sensory perturbation (without sensory perturbations, perturbations of the base of support, visual perturbations, combined sensory perturbations and vestibular perturbations). The SMDs were considered to represent a large difference between groups if the mean exceeded the interval ( -0.8 to 0.8$)$ and if the $95 \%$ CI did not include zero. ${ }^{20}$

The number of patients unable to perform specific balance tasks was converted to percentages as a function of the applied sensory perturbations and graphically presented in a bar chart.

\section{Role of the Funding Source}

The University of Antwerp Research Council (ID33586), the University of Antwerp, and the Antwerp University Hospital supported this work, and C. McCrum was funded by the NUTRIM Graduate Programme from NUTRIM, Maastricht University Medical Center + . The funders played no role in the design, conduct, or reporting of this study.

\section{Results}

\section{Study Selection}

The search query revealed 866 unique citations. After screening, 19 studies met the selection criteria, but 7 studies were excluded after risk of bias assessment. ${ }^{21-27}$ Data were extracted from 12 eligible studies ${ }^{28-39}$ as reference screening revealed no additional suitable citations. The selection process is presented in Supplementary Appendix 2.

\section{Risk of Bias in Individual Studies}

The methodological quality of 8 studies was rated as high $^{28-32,35,38,39}$ and 4 as acceptable..$^{33,34,36,37}$ All studies of high quality failed to compare similarities or differences between participants and nonparticipants but satisfied all other criteria. Studies of acceptable quality failed to provide clarity on including clear definitions of the source population, ${ }^{36}$ addressing the influence of confounding factors, ${ }^{33,36,37}$ applying the same selection criteria for both controls and patients, ${ }^{33,34,36}$ or not comparing similarities or differences between participants and non-participants..$^{33,34,36,37}$ Seven studies were excluded as they did not include clear definitions of the source population ${ }^{21,22,26,27}$ or address the influence of confounding factors. ${ }^{21,23-26}$

\section{Population Characteristics}

Spread over 12 studies, 176 patients with BVP (21-90 years old; 82 males, 85 females) ${ }^{28-39} 196$ patients with UVP (20-92 years old; 56 male, 72 female), ${ }^{28,32,33,39}$ and 205 HC (18-87 years old; 86 male, 112 female $)^{28-31,33-38}$ were investigated. In 8 studies, the control population was age matched to the BVP population..$^{28-32,35,36,38}$

The diagnosis of BVP was established using (a combination of) different vestibular function tests: (1) reduced VOR gain values during rotational chair testing at different frequencies $(0.01-0.8 \mathrm{~Hz})^{29-32,38}$ or at different velocities $\left(47.1^{\circ} / \mathrm{s}-240^{\circ} / \mathrm{s}\right),{ }^{35,36}(2)$ reduced or absent responses to caloric stimulation, ${ }^{28,31-34,36-39}$ (3) corrective saccades or reduced responses to head impulses,,$^{33,34,37}$ or (4) reduced responses to cervical/ocular vestibular-evoked myogenic potentials. ${ }^{33,34}$ Etiology of the vestibular loss was reported in 11 studies and varied greatly across the 176 patients with BVP and 196 patients with UVP: acoustic tumor (UVP $\mathrm{n}=29$ ) ${ }^{28,32,33}$ degeneration $(\mathrm{BVP} \mathrm{n}=3),{ }^{32}$ genetic disorder $(\mathrm{BVP} \mathrm{n}=3),{ }^{34}$ idiopathic (BVP $\mathrm{n}=28$, UVP $\mathrm{n}=14),{ }^{30-33,38}$ inflammation/infection (BVP $\mathrm{n}=17$, UVP $\mathrm{n}=38),{ }^{28-34,36,38}$ injury $(\mathrm{BVP} \mathrm{n}=1),{ }^{34}$ (inner) ear surgery (BVP $\mathrm{n}=1, \mathrm{UVP} \mathrm{n}=28)^{29,32,33}$ Menière's disease (BVP $n=4$, UVP $n=4)$, ${ }^{28,34,36}$ multiple sensory deficit (UVP $\mathrm{n}=5$ ), ${ }^{32}$ ototoxicity (BVP $\mathrm{n}=57$, UVP $\mathrm{n}=2){ }^{29-33,35,36,38}$ unknown (BVP $\mathrm{n}=5$, UVP $\mathrm{n}=34$ ), ${ }^{28,34}$ and not reported (BVP $\mathrm{n}=57$, UVP $\mathrm{n}=42)^{28,37,39}$ Time since BVP onset was reported in 4 studies $^{29,32,35,39}$ and varied between 1 month $^{35}$ and 15 years. ${ }^{29}$

\section{Balance Performance Without Sensory Perturbations}

Differences in performance on balance tasks without sensory perturbations between patients with BVP and healthy controls (HCs) or patients with UVP are summarized in Figure 1. Standing balance was addressed by measuring the center of pressure (COP) displacements during quiet bipedal stance (hereafter referred to as "standing") on a force plate. Reported variables for standing balance tasks were typical COP measures (eg, 
1. Bilateral Vestibulopathy (BVP) versus healthy controls (HCs)

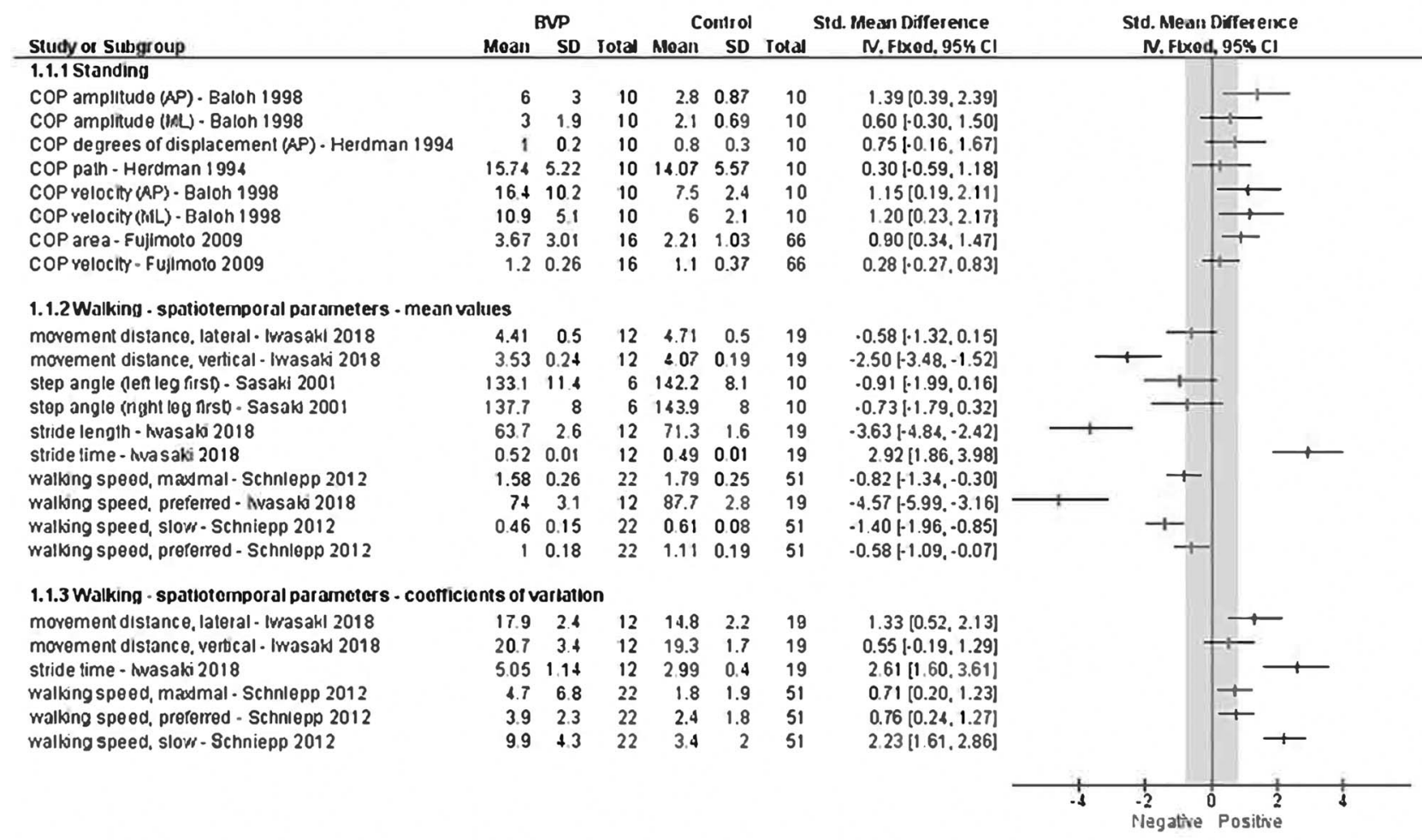

2. Bilateral Vestibulopathy (BVP) versus Unilateral Vestibulopahy (UVP)

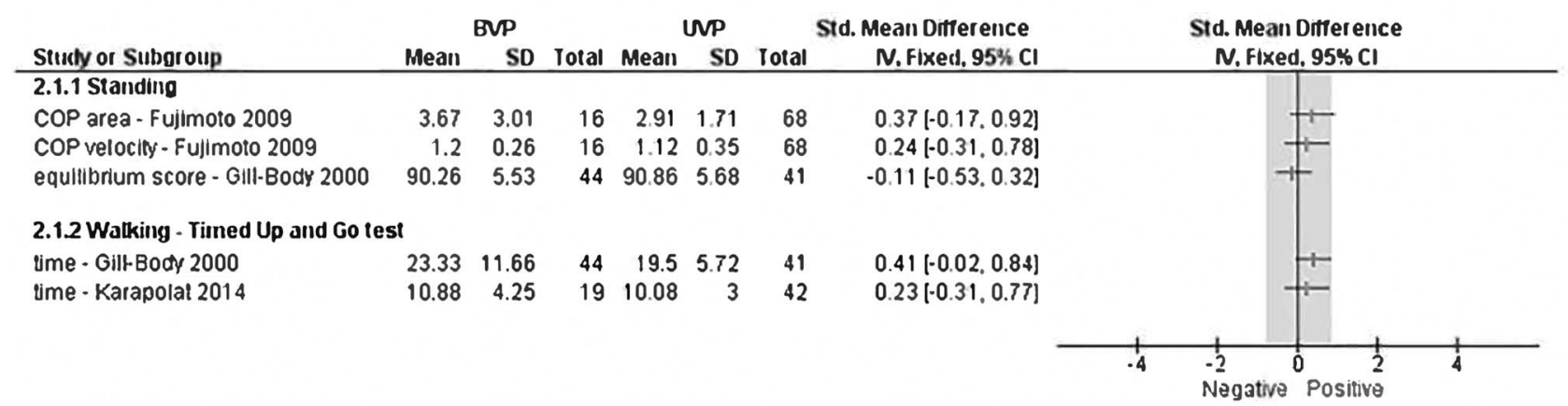

Figure 1.

Difference in performance on balance tasks without sensory perturbations. Data on standing were derived from center of pressure (COP) movements. For walking without sensory perturbation, data were derived from accelerometry ${ }^{34}$, GAITRite ${ }^{37}$, and COP movements ${ }^{36}$ but also timed measures ${ }^{32,39}$. Standardized mean differences were considered to represent a true difference between groups if the difference exceeded the interval ( -0.8 to 0.8$)$ and if the $95 \% \mathrm{Cl}$ did not include zero.

amplitude, area, and velocity $)^{28,30,35}$ but also SD of dynamic alignment (ie, the angular position of the center of gravity relative to the center of foot position) ${ }^{35}$ and the strategy (ie, the relative amounts of ankle and hip movements with lower scores indicating hip strategy). ${ }^{35}$ Comparison of balance performance during standing of patients with BVP and HCs revealed significant SMDs for the COP amplitude in anteroposterior direction (AP), ${ }^{30} \mathrm{COP}$ area, ${ }^{28}$ 
COP velocity AP, and mediolateral direction (ML), ${ }^{30}$ but not for the other variables ${ }^{28,30,35}$ or for comparison between performance of BVP and UVP. ${ }^{28,32}$

Walking balance was assessed with overground walking $^{34,36,37}$ at different walking speeds (slow, ${ }^{37}$ preferred, ${ }^{34,37}$ and maximal ${ }^{37}$ ) and with the TUG test. ${ }^{32,39}$ From overground walking, stride length, ${ }^{34}$ stride time, ${ }^{34}$ walking speed, ${ }^{34,37}$ lateral and vertical center of mass (COM) excursion ${ }^{34}$ (mean and coefficients of variation [CoV]), and step angles ${ }^{36}$ were extracted. With the TUG, time ${ }^{32,39}$ was measured. Significant SMDs were reported between patients with BVP and HCs for lateral COM excursion $\mathrm{CoV} ;{ }^{34}$ vertical COM excursion $;^{34}$ slow, ${ }^{34}$ preferred, ${ }^{33}$ and slow walking speed $(\mathrm{CoV})^{37}$ both overground and during TUG; stride length $;{ }^{34}$ and stride time $(\mathrm{CoV}){ }^{34}$ No significant differences were found between BVP and UVP for TUG performance. ${ }^{32,39}$

\section{Balance Performance With Perturbations of the} Base of Support

Figure 2 depicts the SMD in performances on balance tasks involving proprioceptive perturbations between patients with BVP and HCs or patients with UVP. In Figure 3, the percentage of patients with BVP unable to complete such tasks is shown. Perturbations of the base of support were introduced during standing by adding foam, ${ }^{28,30-32,38}$ a platform moving in $\mathrm{AP},{ }^{29,30,32,35}$ or $\mathrm{ML}^{30,33}$ directions, but also with different foot positions such as 1-leg stance ${ }^{32}$ and tandem stance. ${ }^{32}$ For standing on foam and on a moving platform, a large variety of variables derived from COP displacement were reported (Figs. 2 and 3). Standing on foam resulted in significant mean differences between BVP and HCs in case of bandwidth of COP displacement, ${ }^{31,38}$ COP acceleration, ${ }^{31}$ COP acceleration root mean squared $\mathrm{AP} / \mathrm{ML},{ }^{31} \mathrm{COP}$ amplitude $\mathrm{AP},{ }^{30} \mathrm{COP}$ root mean squared (AP/ML), ${ }^{31}$ and COP velocity ${ }^{28} \mathrm{AP} / \mathrm{ML} .^{30,31}$ When standing on a platform moving in an AP direction, SMDs between patients with BVP and HCs reflected a large difference in performance for all variables, except for contraction time of $\mathrm{mm}$. gastrocnemius and hamstrings when performing a protective stepping response ${ }^{29}$ as well as the COP path $^{35}$ and the applied strategy (predominance of ankle strategy). ${ }^{35}$ A platform moving in the ML direction also induced significantly more sway in BVP compared with HCs. ${ }^{30}$ Compared with UVP, the COP velocity during standing on foam ${ }^{28}$ and the equilibrium score when standing on a moving platform in AP direction ${ }^{32}$ are significantly smaller in patients with BVP.

\section{Balance Performance With Visual Perturbations}

All data from balance tasks with visual perturbations are presented in Figure 4. During standing, eyes closed (EC) and altered visual surround (condition 3 of the sensory organization test) were used to introduce visual perturbations. During these conditions, COP displacements were measured. In the EC condition, compared with HCs, the bandwidth of COP displacement, ${ }^{38} \mathrm{COP}$ amplitude $\mathrm{AP} / \mathrm{ML},{ }^{30} \mathrm{COP}$ degrees of displacement (AP),$^{35} \mathrm{COP}$ velocity $\mathrm{AP} / \mathrm{ML},{ }^{30}$ and the SD of dynamic alignment ${ }^{35}$ differed significantly in patients with BVP. Standing with altered visual surround induced significant SMDs (patients with BVP versus HCs) for the COP degrees of displacement (AP) and ankle (HCs) versus more hip (patients with BVP) strategy. ${ }^{35}$ No significant SMDs were found during standing with EC or altered visual surround conditions when comparing performances of patients with BVP with patients with UVP. ${ }^{28,32}$

Walking with EC induced larger deviations in the step angle of both legs in patients with BVP compared with HCs. ${ }^{36}$

\section{Balance Performance With Vestibular Perturbations}

In 1 study $^{32}$, vestibular perturbations were implemented by adding head turns to an overground walking task. Although patients with BVP performed the task faster than patients with UVP, the SMD was only moderate (Fig. 5).

\section{Balance Performance With More Than One Sensory Perturbation}

Figure 6 depicts differences in performance on balance tasks with combined sensory perturbations between patients with BVP and patients with HCs/UVP.

Combined sensory perturbation was introduced in standing tasks by combining the EC condition with foam, ${ }^{28,30,32,38}$ moving platform in AP direction ${ }^{30,32,33,35}$ or ML direction, ${ }^{30,33}$ and by combining altered visual surround with AP movement of the platform..$^{32,35}$ Several studies reported patients to be unable to complete balance tasks with combined sensory perturbation, , $^{31-33,35,38}$ as shown in Figure 3. Overall, regardless of the measured posturography variable, when they are able to complete the task successfully, the performances of patients with BVP differ significantly from those of HCs. A similar trend was found when comparing patients with BVP with patients with UVP, except in the case of acute UVP. ${ }^{33}$

\section{Discussion}

The aim of this systematic review was to explore measures for balance control most useful to quantify the performances of patients with BVP related to different balance domains. The results revealed that the 12 included studies used different types of balance measures ${ }^{28-39}$ and even when similar assessment tools were applied, different parameters were used. ${ }^{29-31,35}$ The lack of uniformity in the applied methods among studies highlights the tone for a lacuna in the literature with respect to a standardized test battery for balance control in BVP. As a result, this systematic review merely provides a description of results 


\section{Bilateral Vestibulopathy (BVP) versus healthy controls (HCs)}

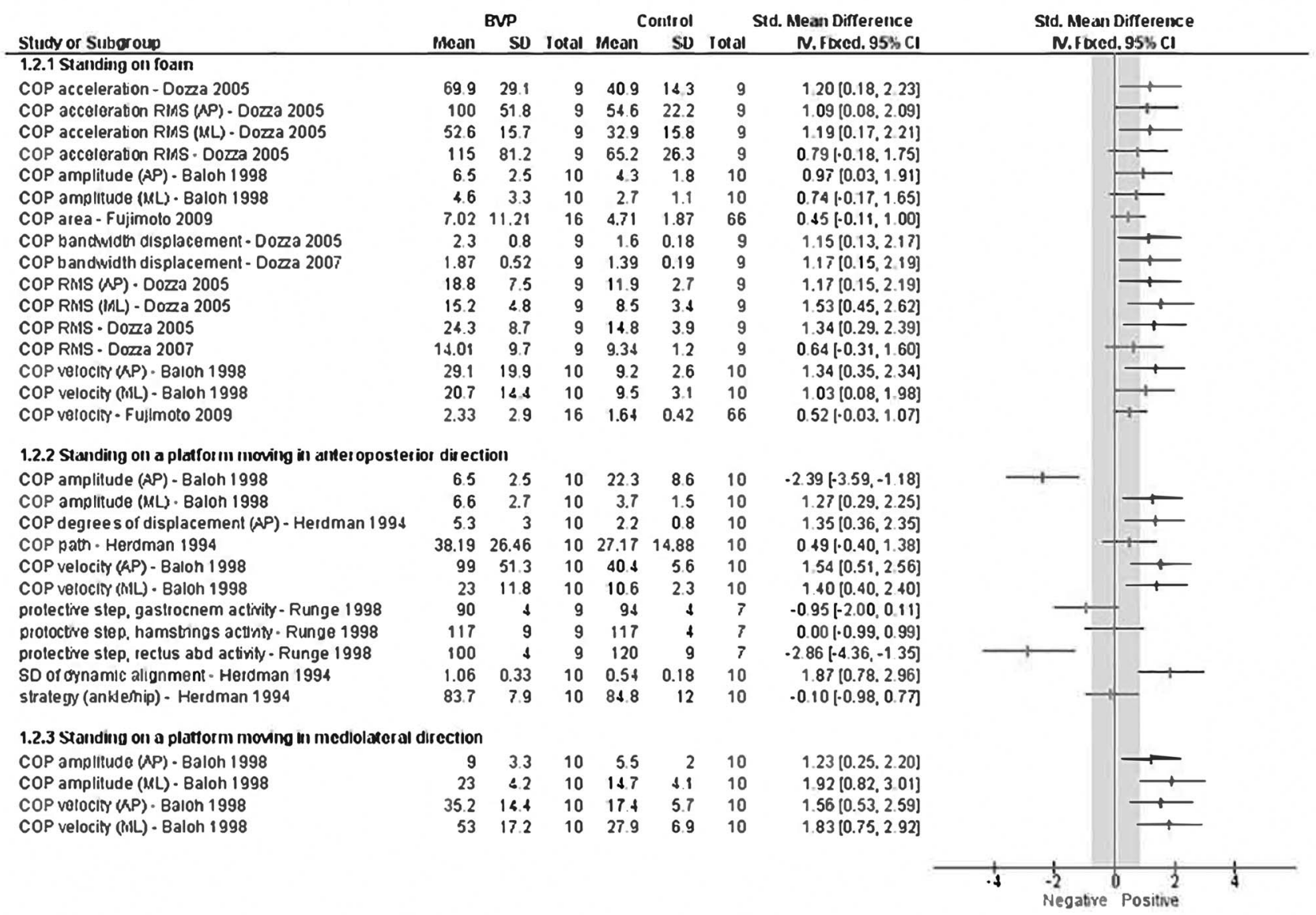

2. Bilateral Vestibulopathy (BVP) versus Unilateral Vestibulopahy (UVP)

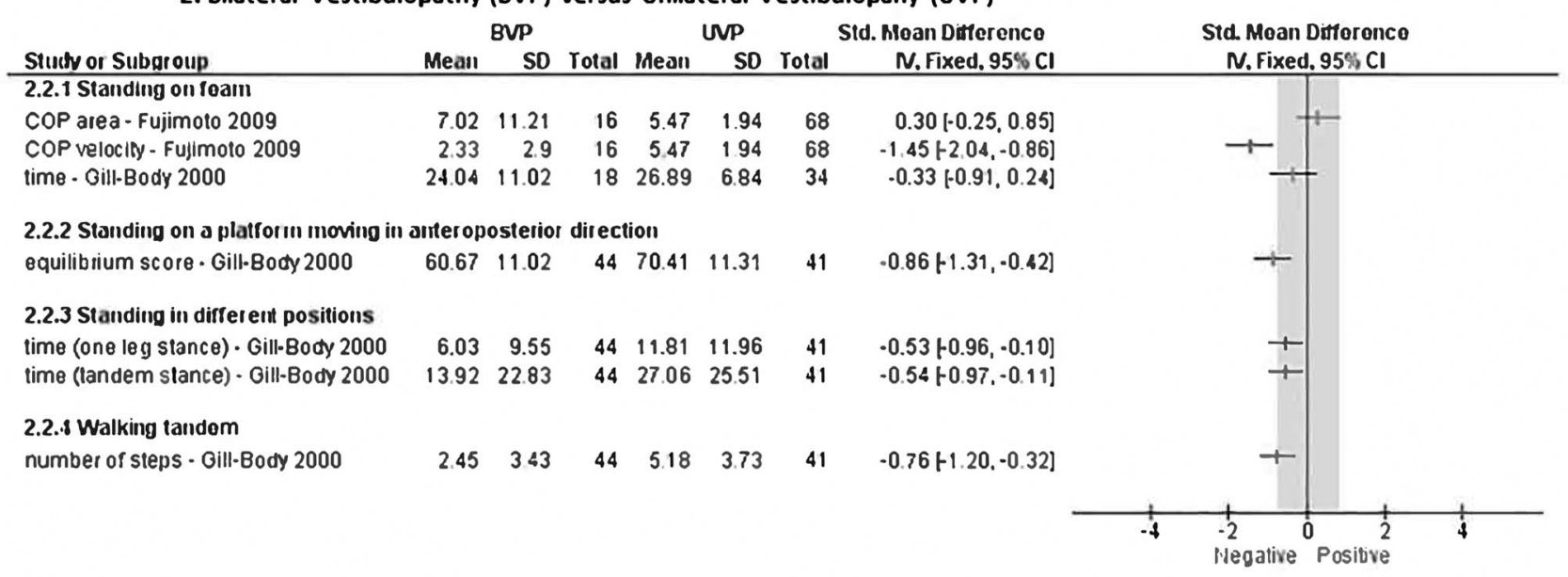

Figure 2.

Difference in performance on balance tasks without proprioceptive perturbations. All data were derived from posturographic measurements, representing movements of the center of pressure (COP) with the exception of muscle activity ${ }^{29}$ and timed measures in different positions and number of steps $^{32}$. Standardized mean differences were considered to represent a true difference between groups if the difference exceeded the interval ( -0.8 to 0.8$)$ and if the $95 \% \mathrm{Cl}$ did not include zero. 


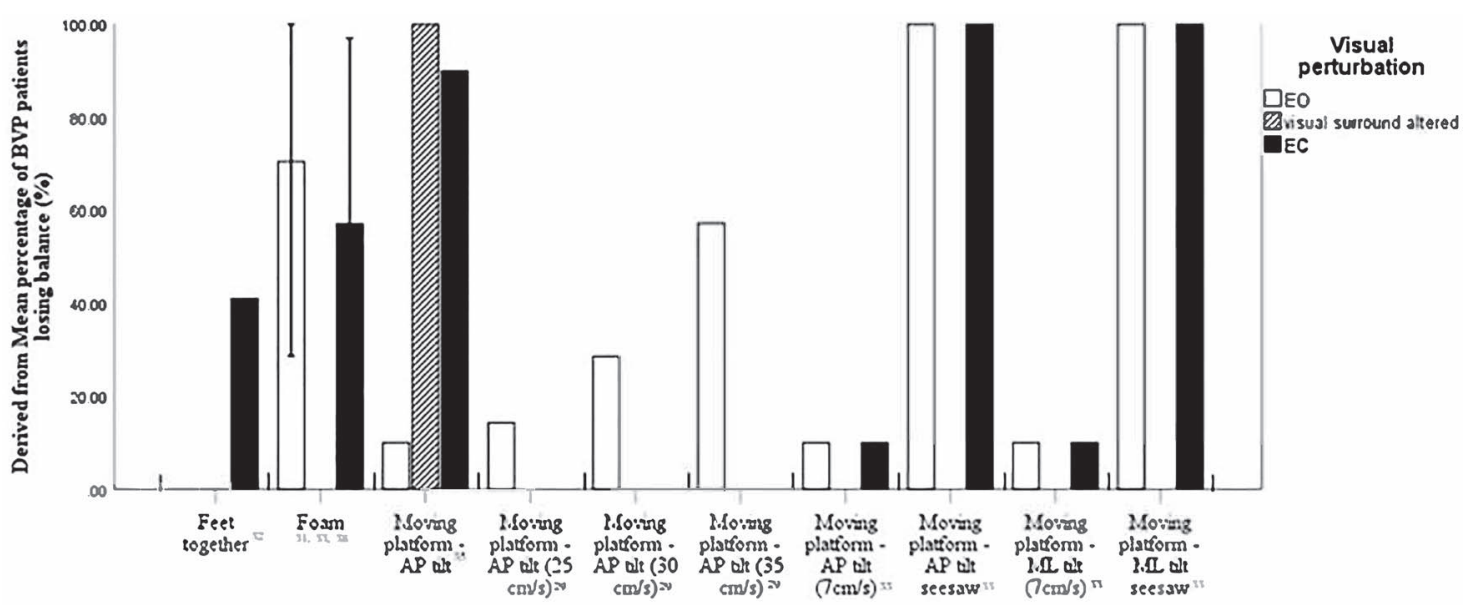

Figure 3.

Mean percentage of patients with bilateral vestibulopathy (BVP) losing balance, classified according to sensory perturbation and balance tasks.

as opposed to a more robust meta-analysis. Nevertheless, some trends can be derived from the available results: (1) when no sensory perturbations were present (ie, balance tasks with natural base of support on stable ground with eyes open), the performances of patients with BVP were similar to those of patients with UVP and, for the most part, also to those of HCs; (2) when only perturbations of the base of support or visual perturbations were present, patients with BVP showed an increase in postural sway compared with HCs, whereas the differences varied (mean SMD: $[|0.01| ;|1.45|]$ ) strongly compared with patients with UVP; (3) the presence of more than 1 sensory perturbation led to outcomes that clearly distinguished patients with BVP from both HCs and patients with UVP shown by large mean differences (SMD $\geq 0.80$ ) in almost all outcomes (standing on moving platform/foam with EC or tandem gait with EC).

These results are not surprising. In any perturbed sensory situation, patients with BVP can rely on only 1 sensory source. However, which test should be preferred by clinicians to identify these patients in clinical practice and subsequently evaluate treatment effects?

Using the Romberg test, as was suggested by the Bárány Society, ${ }^{5}$ for the assessment of balance has several limitations. One author used the Romberg test with EC and found a medium SMD between patients with BVP and patients with UVP (SMD $=-0.58,95 \% \mathrm{CI}=[-1.17$ to 0.00$]$ ). ${ }^{32}$ The Romberg test does not take into account the uneven terrain, which is a typical area of difficulty in patients with BVP. ${ }^{5}$ Therefore, more challenging test protocols that do take these patient's complaints into account seem more suitable.
Technical measures, such as the Sensory Organization Test, are much more challenging and provide us with valuable information regarding the patient's ability to reweight sensory information, ${ }^{40}$ which is clearly disturbed in patients with BVP (Figs. 1-6). When using a moving platform, either in anteroposterior or mediolateral direction, large differences can be observed between patients with BVP and HCs depending on the outcome parameter $^{29,30,35}$ as well as medium mean differences compared with patients with UVP. ${ }^{32}$ Furthermore, patients with BVP do not only perform poorer quantitatively (eg, time or COP displacement) but also with regard to being unable to achieve, maintain, or restore a specific position in more complex sensory situations (Fig. 3).

In common clinical settings, such technical equipment is not available, underlining the need for more simple substitutes such as foam pads or applying more complex foot positions that seem to suffice when aiming to evaluate patients with BVP (Figs. 2 and 5). Using these task constraints, timing of performance can be useful, but also assessing the (in)ability to achieve and maintain the position (pass/fail) can be useful.

Patients with BVP do not only report balance difficulties in static situations but also in more dynamic situations. ${ }^{5}$ However, fewer included studies have focused on the balance abilities of patients with BVP during walking. . $^{34,36,37,39}$ In contrast to the included literature on standing balance, gait assessment in these patients is scarce. Compared with HCs, the performance of patients with BVP on the Functional Gait Assessment were significantly poorer. ${ }^{37}$ The TUG test did not reveal significant differences between patients with BVP and UVP,$^{32}$ and similar performances on the Dynamic Gait 
1. Bilateral Vestibulopathy (BVP) versus healthy controls (HCs)

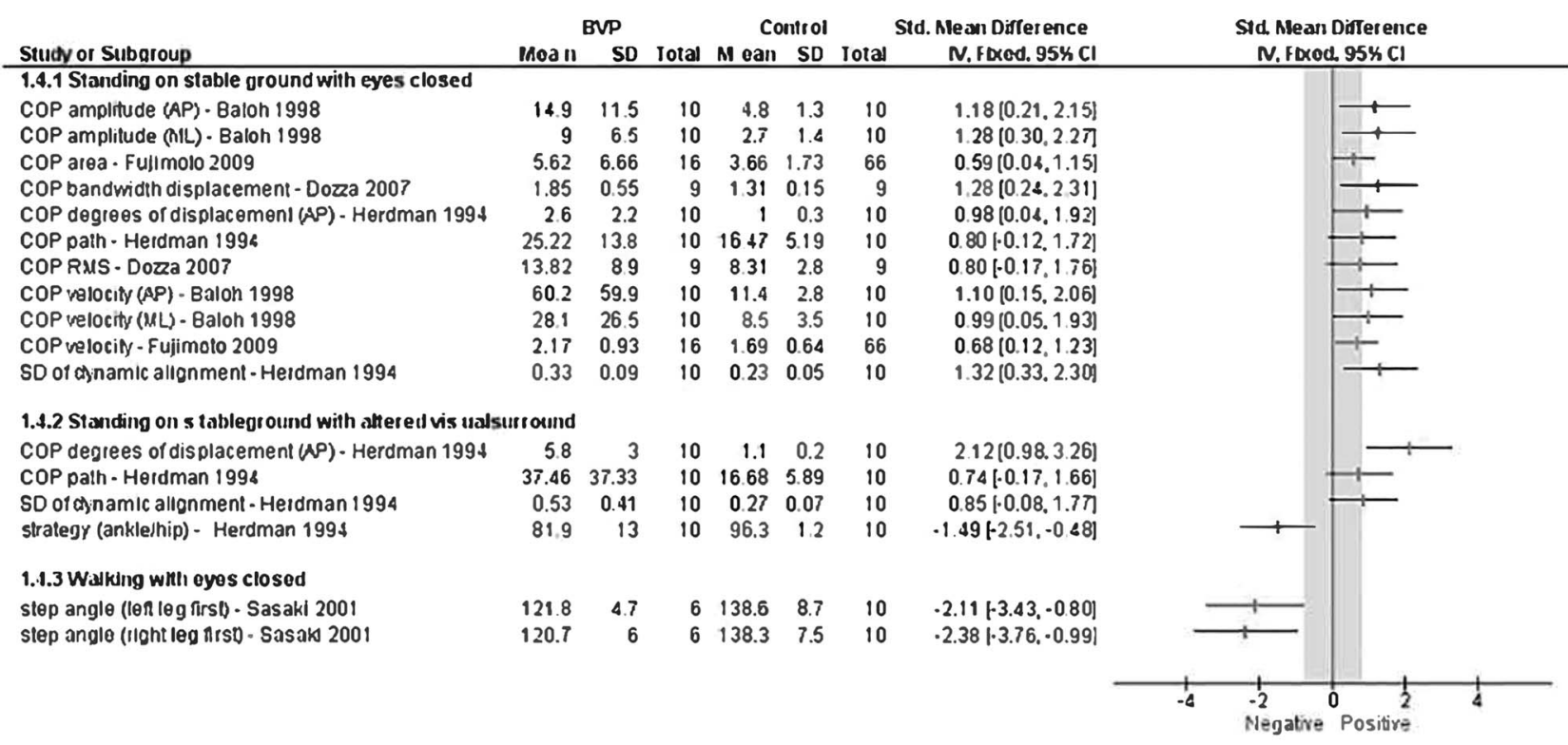

2. Bilateral Vestibulopathy (BVP) versus Unilateral Vestibulopahy (UVP)

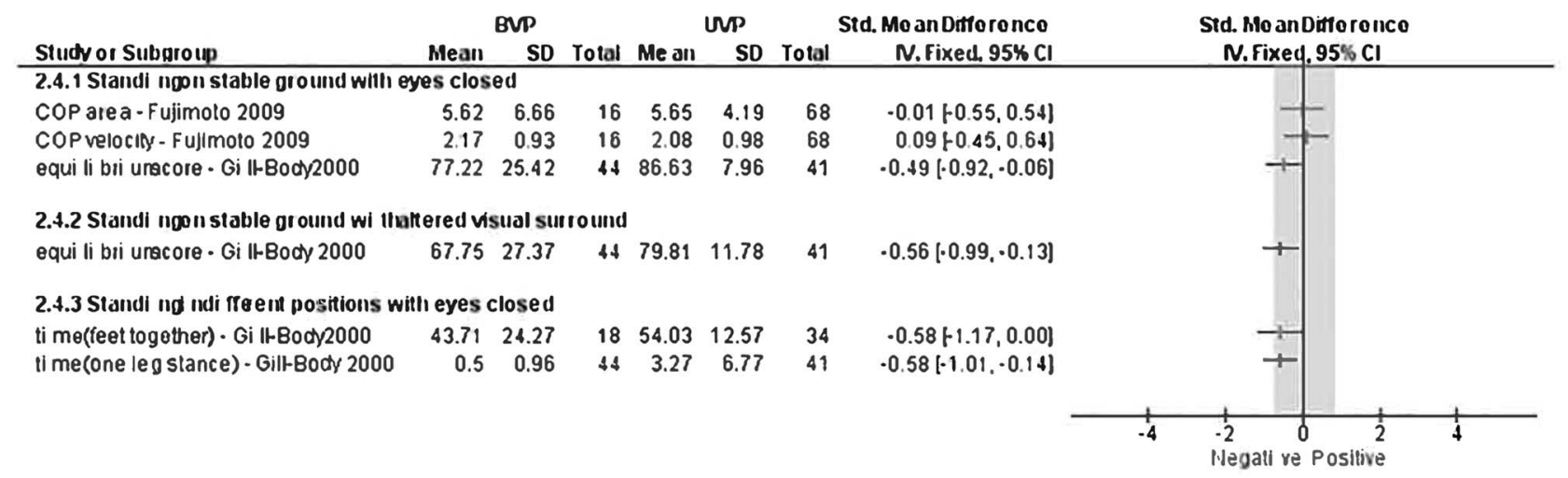

Figure 4.

Difference in performance on balance tasks with visual perturbations. All data were derived from center of pressure (COP) movements except for timed measures in different positions. Standardized mean differences were considered to represent a true difference between groups if the difference exceeded the interval $(-0.8$ to 0.8$)$ and if the $95 \% \mathrm{Cl}$ did not include zero.

\section{Bilateral Vestibulopathy (BVP) versus Unilateral Vestibulopahy (UVP)}

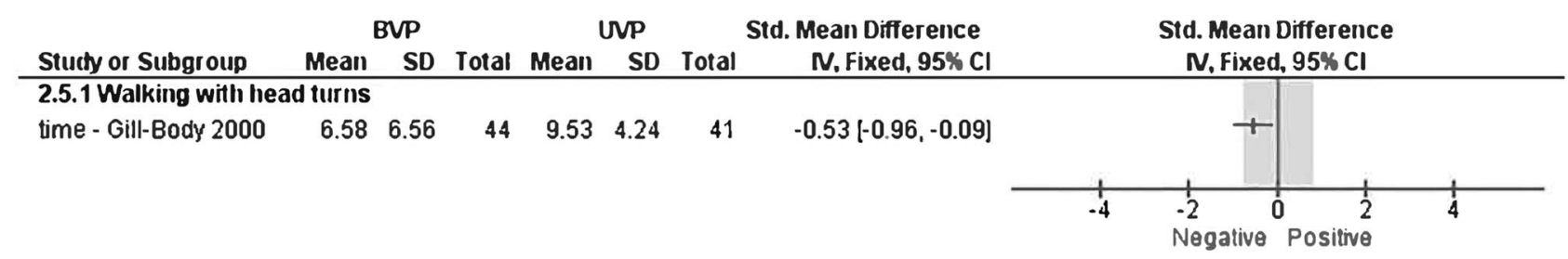

\section{Figure 5.}

Difference in performance on balance tasks with vestibular perturbations. Standardized mean differences were considered to represent a true difference between groups if the difference exceeded the interval $(-0.8$ to 0.8$)$ and if the $95 \% \mathrm{Cl}$ did not include zero. 


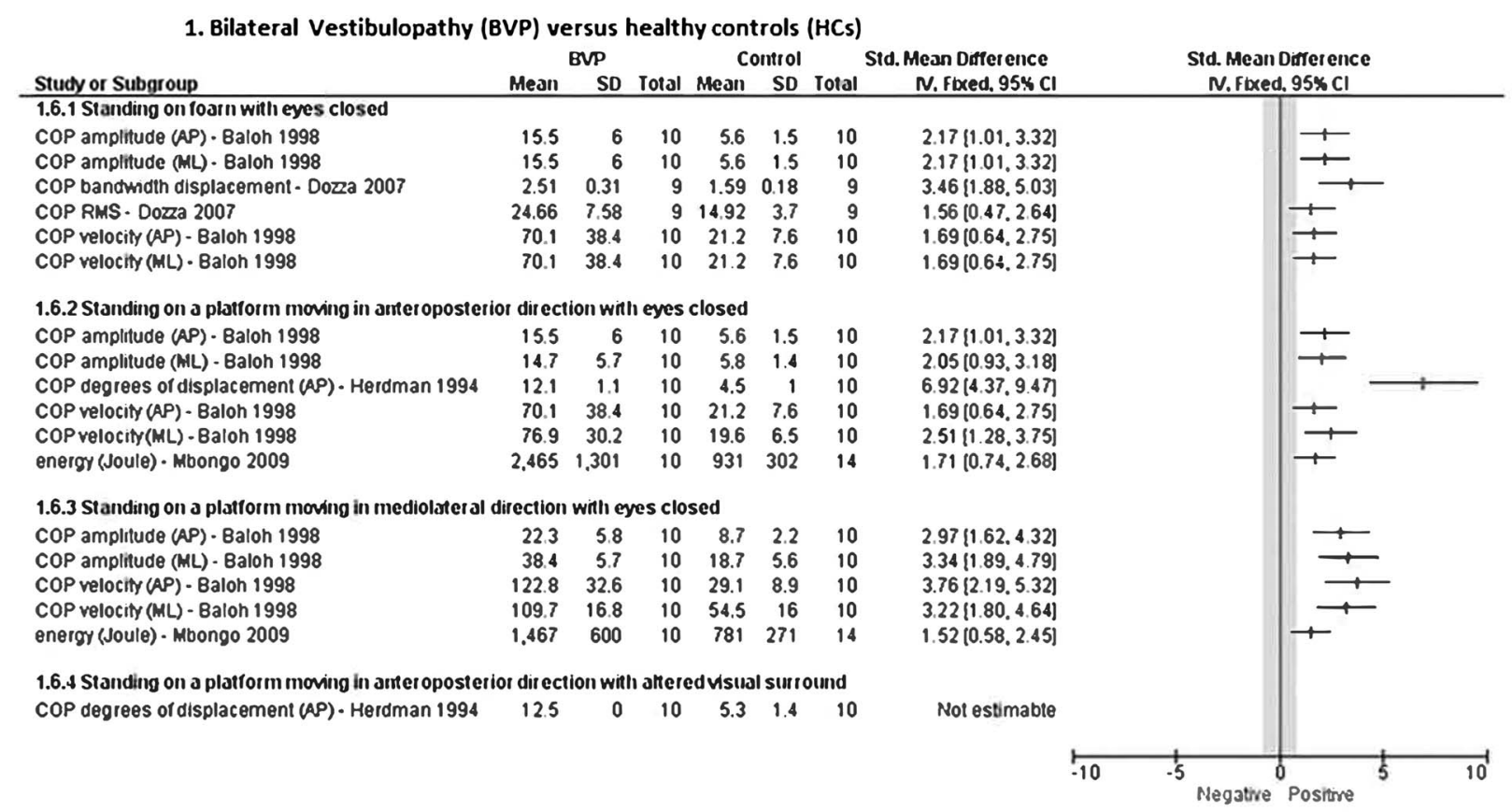

2. Bilateral Vestibulopathy (BVP) versus Unilateral Vestibulopahy (UVP)

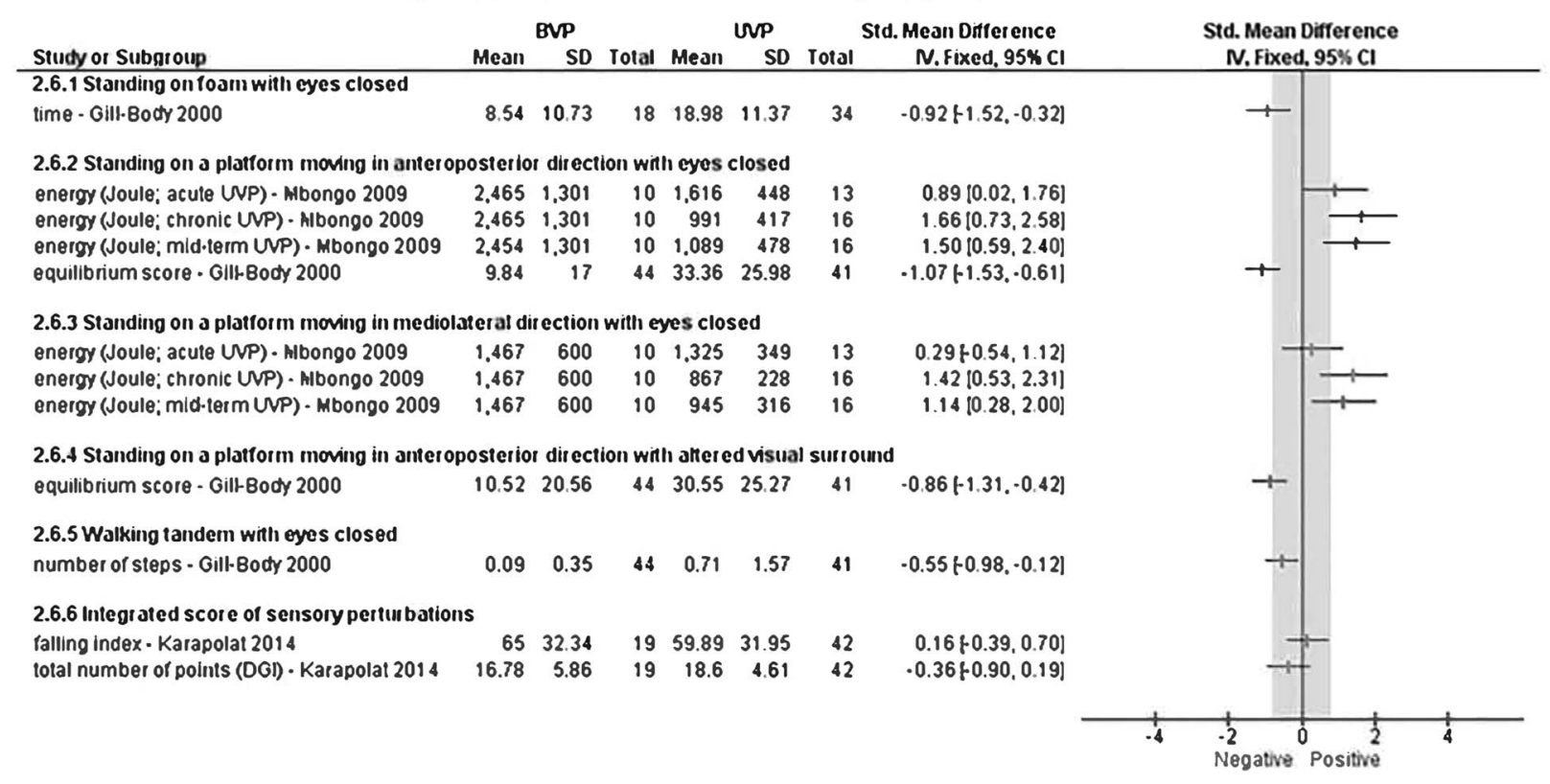

Figure 6.

Difference in performance on balance tasks with combined sensory perturbations. All data were derived from posturographic measurements, representing movements of the center of pressure (COP) with the exception of timed measures in different positions ${ }^{32}$, the falling index during the sensory orientation test ${ }^{39}$, and the total number of points on the Dynamic Gait Index ${ }^{39}$. Standardized mean differences were considered to represent a true difference between groups if the difference exceeded the interval $(-0.8$ to 0.8$)$ and if the $95 \% \mathrm{Cl}$ did not include zero. 


\section{Balance Performance in BVP}

Index between these 2 groups were found. ${ }^{39}$ Walking performances seem to be more dependent on both the applied outcome parameter as the specific walking task under consideration (Fig. 1). Walking requires control of the center of mass when the base of support is changing, thereby requiring more real-time adaptations, ${ }^{41}$ that is, variability of gait, compared with standing conditions. Thus, walking allows more compensatory behavior to maintain control over the body but also requires the availability of compensatory strategies. Therefore, with respect to dynamic balance control, more thorough investigations towards sensitive functional assessment tools are required.

\section{Clinical Implications}

The results of this systematic review indicate that patients with BVP substitute vestibular loss with a combination of proprioceptive and visual cues, allowing adequate compensation for the missing vestibular information. As a result, the balance control of these patients in clinical settings may not be adequately challenged using a single sensory perturbation. To enhance the identification of balance deficits that approximate the subjective complaints of patients with BVP, more advanced and challenging balance tasks need to be performed, that is, involving multiple sensory perturbations. However, the concept of balance control is not only influenced by sensory strategies; patients with BVP also perform poorly on movement strategies (eg, reactive balance control),,$^{30,32,33,35}$ control of dynamics (eg, gait), ${ }^{32,34,36,37}$ orientation in space (eg, perception of verticality), ${ }^{42}$ and cognitive processing (eg, attention). ${ }^{43}$ This should therefore also be implemented in the assessment protocol for addressing balance control in patients with BVP, for which the Balance Evaluations Test ${ }^{44}$ might be suitable. Hence, further investigations towards validity and sensitivity as well as reliability in patients with BVP are useful.

\section{Limitations of the Included Studies}

In general, the studies discussed included a heterogeneous group of participants (ie, patients with BVP of different etiologies), possibly different degrees of vestibular dysfunction, and varying times of onset, which were often $(n=8 / 12)$ not reported. Therefore, findings in this review should be interpreted with caution.

Additionally, the literature search revealed 19 potentially relevant studies to answer the research question, of which 7 studies had to be rejected due to severe methodological shortcomings. Of the 12 eligible studies, only 2 studies $^{32,39}$ compared patients with BVP and UVP and only 2 studies $^{28,33}$ compared BVP with both UVP and HCs. Although most included studies were rated as high quality, 33\% reached only acceptable quality, downgrading the body of evidence due to (1) methodological shortcomings in defining, selecting, or comparing the investigated groups, ${ }^{33,34,36,37}$ (2) inducing selection bias and lacking identification of confounding factors, ${ }^{33,34,36,37}$ and (3) limiting the reliability of the currently available data.

Related to the controls, only in 8 included studies were controls age-matched to the BVP population, resulting in a possible selection bias. ${ }^{28-32,35,36,38}$ Only few included studies matched the criteria proposed by the Bárány Society because almost all studies included were published before the diagnostic criteria were released. ${ }^{5}$ Most similarities were related to the reduced caloric responses $^{31,33,37,38}$ and reduced bilateral horizontal angular VOR gain. ${ }^{33}$ Only 2 studies clearly stated that participants reported unsteadiness while walking. ${ }^{30,32}$ In the remaining citations, it was unclear whether self-reported unsteadiness during standing or walking was used as an inclusion criterion. The presence of oscillopsia and absence of symptoms while sitting or lying down were never reported as inclusion criteria.

Another main limitation of the included literature, unaddressed with methodological quality assessment, is reporting the etiology and the time since BVP onset. In $35 \%$ of the included patients with BVP, etiology was either not reported or unknown. The wide variety of etiologies that were reported, ranging from injuries to genetic disorders, may further influence balance performance. Only 4 studies ${ }^{29,32,35,39}$ reported the interval between onset of the symptomatology and study date, with the majority including patients with chronic BVP. One study reporting balance performance in acute patients with $\mathrm{BVP}^{35}$ shows that this subgroup tends to perform significantly poorer compared with patients with chronic BVP (Figs. 1, 2, 4, and 6), suggesting the need for subclassification within this patient group when assessing balance control in future research. Finally, most literature available concerns standing balance in different sensory conditions, providing insights into how patients with BVP are able to cope with altered sensory information. However, associations with locomotion-incorporating activities from daily life are still missing. In the clinical practice guidelines for patients with vestibular hypofunction by the Academy of Neurologic Physical Therapy ${ }^{45}$ several balance measures incorporating locomotion activities from daily life, such as the Functional Gait Assessment and Dynamic Gait Index, are recommended. Notwithstanding, there was little mention of those balance measures in the included literature. Only 3 studies mentioned the use of the Functional Gait Assessment ${ }^{37}$ or Dynamic Gait Index, ${ }^{39,46}$ of which 1 study was excluded based on methodological quality ${ }^{46}$ and 1 study did not report SDs, ${ }^{37}$ which made it impossible to calculate the SMD. This was rather surprising as these balance measures are widely used in clinical practice.

Even though SMDs were calculated to provide a straightforward interpretation of the actual differences in balance performance between patients with BVP and 
controls, pooling of results was not possible because of the large variability of applied balance tasks and outcome variables. Therefore, these results can only describe a certain trend in balance performance in patients with BVP, highlighting the need for further research on this topic and to define uniform outcome measures to evaluate balance performance in BVP.

\section{Conclusion}

Based on the data presented in this systematic review, in general, patients with BVP seem to be able to perform on the same level as patients with UVP when visual cues, proprioceptive cues, or a combination of both visual and proprioceptive cues are available. In contrast, when both visual and proprioceptive information is lacking, patients with BVP are unable to perform at the same level as HCs and patients with UVP, except when compared with patients with acute UVP. During clinical assessment to identify balance deficits in BVP or evaluate their therapy progress, balance tasks involving multi-sensory perturbations need to be implemented in the test protocol. Future research should also be directed towards the implementation of balance measures assessing balance control in a multifactorial way that relates to activities of daily life.

\section{Author Contributions}

Concept/idea/research design: N. Herssens, E. Verbecque,

C. McCrum, K. Meijer, R. van de Berg, W. Saeys, L. Vereeck, V. Van Rompaey, A. Hallemans

Writing: N. Herssens, E. Verbecque, C. McCrum, R. van de Berg, L. Vereeck, A. Hallemans

Data collection: N. Herssens, E. Verbecque

Data analysis: N. Herssens, E. Verbecque

Project management: N. Herssens, E. Verbecque

Fund procurement: A. Hallemans, K. Meijer

Providing institutional liaisons: R. van de Berg, V. Van Rompaey

Consultation (including review of manuscript before submitting):

C. McCrum, K. Meijer, R. van de Berg, W. Saeys, L. Vereeck,

V. Van Rompaey, A. Hallemans

$N$. Herssens and E. Verbecque are joint first authors. They contributed equally to the conception of the study, analysis, and interpretation of the data and drafting of the manuscript.

\section{Ethics Approval}

None.

\section{Funding}

This work was supported by the University of Antwerp Research Council (grant number ID33586), the University of Antwerp, and the Antwerp University Hospital. C.M. was funded by the NUTRIM
Graduate Programme from NUTRIM, Maastricht University Medical Center+.

\section{Systematic Review Registration}

This systematic review was registered with the International Prospective Register of Systematic Reviews (ref. no. CRD42018102354; www.crd.york.ac.uk/prospero). This review was conducted according to the Preferred Reporting Items for Systematic Review and Meta-Analysis Statement.

\section{Disclosures and Presentations}

The authors completed the ICMJE Form for Disclosure of Potential Conflicts of Interest and reported no conflicts of interest.

DOI: $10.1093 /$ ptj/pzaa083

\section{References}

1 Ward BK, Agrawal Y, Hoffman HJ, Carey JP, Della Santina CC. Prevalence and impact of bilateral vestibular hypofunction: results from the 2008 US National Health Interview Survey. JAMA Otolaryngol Head Neck Surg. 2013;139:803-810.

2 Lucieer F, Duijn S, Van Rompaey VV, et al. Full spectrum of reported symptoms of bilateral vestibulopathy needs further investigation-a systematic review. Front Neurol. 2018;9:352.

3 Hain TC, Cherchi M, Yacovino DA. Bilateral vestibular loss. Semin Neurol. 2013;33:195-203.

4 Lucieer F, Vonk P, Guinand N, Stokroos R, Kingma H, van de Berg R. Bilateral vestibular hypofunction: insights in etiologies, clinical subtypes, and diagnostics. Front Neurol. 2016;7:26.

5 Strupp M, Kim JS, Murofushi T, et al. Bilateral vestibulopathy: diagnostic criteria consensus document of the classification Committee of the Barany Society. J Vestibul Res-Equil. 2017; 27:177-189.

6 Fraker NBM, Ford-Smith C, Wallace B, Fell DW. Chapter 8, Examination and Evaluation of vestibular function, in: Fell DW, Lunnen KY, Rauk RP. A patient-centered approach from examination to interventions and outcomes. F.A. Davis Company: LIFESPAN NEUROREHABILITATION; 2018.

7 Horak FB. Postural orientation and equilibrium: what do we need to know about neural control of balance to prevent falls. Age Ageing. 2006;35:ii7-ii11.

8 Shumway-Cook A. Woollacott MH. Translating Research Into Clinical Practice. Philadelphia, PA: Lippincott Williams \& Wilkins: Motor Control; 2007.

9 Peterka RJ. Chapter 2, Sensory integration for human balance control. In: Day BL, Lord SR, eds. Handb Clin Neurol. Vol 159 (3rd series) Balance, Gait, and Falls.

10 Kim S, Oh YM, Koo JW, Kim JS. Bilateral vestibulopathy: clinical characteristics and diagnostic criteria. Otol Neurotol. 2011;32:812-817.

11 van der Kooij H, Peterka RJ. Non-linear stimulus-response behavior of the human stance control system is predicted by optimization of a system with sensory and motor noise. $J$ Comput Neurosci. 2011;30:759-778.

12 Ernst MO, Banks MS. Humans integrate visual and haptic information in a statistically optimal fashion. Nature. 2002;415:429-433.

13 Lacour M, Helmchen C, Vidal PP. Vestibular compensation: the neuro-otologist's best friend. J Neurol. 2016;263:S54-S64.

14 Lacour M, Bernard-Demanze L. Interaction between vestibular compensation mechanisms and vestibular rehabilitation therapy: 10 recommendations for optimal functional recovery. Front Neurol. 2014;5:285. 
15 Zacharias GL, Young LR. Influence of combined visual and vestibular cues on human perception and control of horizontal rotation. Exp Brain Res. 1981;41:159-171.

16 Mergner T, Becker W. Perception of horizontal self-rotation: Multisensory and cognitive aspects. In: Warren R, Wertheim $\mathrm{AH}$, eds., Perception and Control of Self-Motion. Resources for Ecological Psychology Series). Mahwah, NJ, USA: Lawrence Erlbaum Associates; 1990: 219-263.

17 Hillier S, Inglis-Jassiem G. Rehabilitation for community-dwelling people with stroke: home or centre based? A systematic review. Int J Stroke. 2010;5:178-186.

18 Eysel-Gosepath K, McCrum C, Epro G, Bruggemann GP, Karamanidis $K$. Visual and proprioceptive contributions to postural control of upright stance in unilateral vestibulopathy. Somatosens Mot Res. 2016;33:72-78.

19 Scottish Intercollegiate Guidelines Network SIGN. https:// www.sign.ac.uk/checklists-and-notes.html. Accessed October 9, 2019.

20 Lee DK. Alternatives to P value: confidence interval and effect size. Korean J Anesthesiol. 2016;69:555-562.

21 Kapoula Z, Gaertner C, Yang Q, Denise P, Toupet M. Vergence and standing balance in subjects with idiopathic bilateral loss of vestibular function. Plos One. 2013;8:e66652.

22 Suarez H, Sotta G, San Roman C, et al. Postural response characterization in elderly patients with bilateral vestibular hypofunction. Acta Otolaryngol. 2013;133:361-367.

23 McGibbon CA, Krebs DE, Scarborough DM. Vestibulopathy and age effects on head stability during chair rise. Acta Otolaryngol. 2001;121:52-58.

24 Mamoto Y, Yamamoto K, Imai T, Tamura M, Kubo T. Three-dimensional analysis of human locomotion in normal subjects and patients with vestibular deficiency. Acta Otolaryngol. 2002;122:495-500.

25 Cavanaugh JT, Goldvasser D, McGibbon CA, Krebs DE. Comparison of head- and body-velocity trajectories during locomotion among healthy and vestibulopathic subjects. $J$ Rehabil Res Dev. 2005;42:191-198.

26 Guigou C, Toupet M, Delemps B, Heuschen S, Aho S, Bozorg GA. Effect of rotating auditory scene on postural control in normal subjects, patients with bilateral vestibulopathy, unilateral, or bilateral cochlear implants. Front Neurol. 2018;9:972.

27 Lacour M, Dosso NY, Heuschen S, Thiry A, Van Nechel C, Toupet M. How eye movements stabilize posture in patients with bilateral vestibular hypofunction. Front Neurol. 2018;9:744.

28 Fujimoto C, Murofushi T, Chihara Y, et al. Assessment of diagnostic accuracy of foam posturography for peripheral vestibular disorders: analysis of parameters related to visual and somatosensory dependence. Clin Neurophysiol. 2009;120:1408-1414.

29 Runge CF, Shupert CL, Horak FB, Zajac FE. Role of vestibular information in initiation of rapid postural responses. Exp Brain Res. 1998;122:403-412.

30 Baloh RW, Jacobson KM, Beykirch K, Honrubia V. Static and dynamic posturography in patients with vestibular and cerebellar lesions. Arch Neurol. 1998;55:649-654.
31 Dozza M, Chiari L, Horak FB. Audio-biofeedback improves balance in patients with bilateral vestibular loss. Arch Phys Med Rehabil. 2005;86:1401-1403.

32 Gill-Body KM, Beninato M, Krebs DE. Relationship among balance impairments, functional performance, and disability in people with peripheral vestibular hypofunction. Phys Ther. 2000;80:748-758.

33 Mbongo F, Qu'hen C, Vidal PP, Tran Ba Huy P, De Waele C. Role of vestibular input in triggering and modulating postural responses in unilateral and bilateral vestibular loss patients. Audiol Neurootol. 2009;14:130-138.

34 Iwasaki S, Fujimoto C, Egami N, et al. Noisy vestibular stimulation increases gait speed in normals and in bilateral vestibulopathy. Brain Stimul. 2018;11:709-715.

35 Herdman SJ, Sandusky AL, Hain TC, Zee DS, Tusa RJ. Characteristics of postural stability in patients with aminoglycoside toxicity. J Vestib Res. 1994;4:71-80.

36 Sasaki O, Asawa S, Katsuno S, Usami S, Taguchi K. Gait initiation in bilateral vestibular loss. Auris Nasus Larynx. 2001;28:295-299.

37 Schniepp R, Wuehr M, Neuhaeusser M, et al. Locomotion speed determines gait variability in cerebellar ataxia and vestibular failure. Mov Disord. 2012;27:125-131.

38 Dozza M, Horak FB, Chiari L. Auditory biofeedback substitutes for loss of sensory information in maintaining stance. Exp Brain Res. 2007;178:37-48.

39 Karapolat H, Celebisoy N, Kirazli Y, et al. Is vestibular rehabilitation as effective in bilateral vestibular dysfunction as in unilateral vestibular dysfunction? Eur J Phys Rehabil Med. 2014;50:657-663.

40 Nashner LM, Black FO, Wall C 3rd. Adaptation to altered support and visual conditions during stance: patients with vestibular deficits. J Neurosci. 1982;2:536-544.

41 Bruijn SM, JHv D. Control of human gait stability through foot placement. J R Soc Interface. 2018;15:20170816.

42 Toupet M, Van Nechel C, Hautefort C, Duquesne U, Heuschen S, Bozorg GA. Subjective visual vertical in idiopathic bilateral vestibular hypofunction: enhanced role of vision, neck, and Body proprioception. Otol Neurotol. 2017;38:1010-1016.

43 Dobbels B, Peetermans O, Boon B, Mertens G, Van de Heyning P, Van Rompaey V. Impact of bilateral vestibulopathy on spatial and nonspatial cognition: a systematic review. Ear Hear. 2019;40:757-765.

44 Horak FB, Wrisley DM, Frank J. The balance evaluation systems test (BESTest) to differentiate balance deficits. Phys Ther. 2009;89:484-498.

45 Hall CD, Herdman SJ, Whitney SL, et al. Vestibular rehabilitation for peripheral vestibular hypofunction: an evidence-based clinical practice guideline: from the American Physical Therapy Association Neurology Section. J Neurol Phys Ther. 2016;40:124-155.

46 Herdman SJ, Hall CD, Maloney B, Knight S, Ebert M, Lowe J. Variables associated with outcome in patients with bilateral vestibular hypofunction: preliminary study.J Vestib Res. 2015;25:185-194. 